\title{
ACAROLOGY
}

\section{Pode Euseius alatus DeLeon (Acari: Phytoseiidae) Predar Aceria guerreronis Keifer (Acari: Eriophyidae) em Coqueiro?}

\author{
José W da S Melo ${ }^{1}$, Cleiton A Domingos ${ }^{1}$, Manoel G C Gondim JR ${ }^{1}$, Gilberto J de Moraes² \\ ${ }^{1}$ Área de Fitossanidade, Depto. Agronomia, Univ. Federal Rural de Pernambuco, Rua Dom Manoel de Medeiros, s/n \\ 52171-900, Recife, PE; mguedes@depa.ufrpe.br; ${ }^{2}$ Depto. Entomologia, Fitopatologia e Zoologia Agrícola, Escola \\ Superior de Agricultura “Luiz de Queiroz”, USP, Av. Pádua Dias, 11, C. postal 9, 13418-900, Piracicaba, SP; \\ gjmoraes@carpa.ciagri.usp.br
}

Edited by Denise Návia - EMBRAPA

Neotropical Entomology 38(1):139-143 (2009)

Can Euseius alatus DeLeon (Acari: Phytoseiidae) Prey on Aceria guerreronis Keifer (Acari: Eriophyidae) in Coconut Palm?

\begin{abstract}
Mites of the genus Euseius are generally considered specialist as pollen feeders. Euseius alatus DeLeon is one of the six species of phytoseiid mites most commonly found on coconut plants in northeast Brazil associated with Aceria guerreronis Keifer. Although the morphology of E. alatus does not favor the exploitation of the meristematic area of the fruit inhabited by A. guerreronis, the predator may have some role in the control of this eriophyid during the dispersion process. The objective of this work was to evaluate the development and reproduction of E. alatus on the following diets: $A$. guerreronis, Ricinus communis pollen (Euphorbiaceae), and Tetranychus urticae Koch (Tetranychidae) $+R$. communis pollen + honey solution $10 \%$. Euseius alatus developed slightly faster and had slightly higher oviposition rate when feeding on the diet composed of T. urticae + pollen + honey. However, life table parameters were very similar on all diets, suggesting that $E$. alatus may contribute in reducing the population of $A$. guerreronis in the field.
\end{abstract}

KEY WORD: Biology, mite, predator, pollen feeding mite, coconut mite, phytoseiid

RESUMO - Ácaros do gênero Euseius são geralmente considerados especialistas na alimentação de pólen. Euseius alatus DeLeon é uma das seis espécies de ácaros fitoseídeos mais comumente encontrados em plantas de coqueiro no Nordeste do Brasil, associado com Aceria guerreronis Keifer. Apesar de a morfologia de E. alatus não favorecer a exploração da área meristemática do fruto habitada por A. guerreronis, o predador pode ter algum papel no controle do eriofídeo durante o processo de dispersão. O objetivo deste trabalho foi avaliar o desenvolvimento e a reprodução de E. alatus nas seguintes dietas: A. guerreronis, pólen de Ricinus communis (Euphorbiaceae); e Tetranychus urticae Koch (Tetranychidae) + pólen de R. communis + mel a $10 \%$. Euseius alatus desenvolveu-se mais rapidamente e ovipositou mais quando alimentada em dieta composta por T. urticae + pólen + mel. Contudo, os parâmetros da tabela de vida foram muito semelhantes em todas as dietas, sugerindo que E. alatus pode contribuir na redução da população de A. guerreronis no campo.

PALAVRAS-CHAVE: Biologia, ácaro, predador, ácaro que se alimenta de pólen, ácaro do coqueiro, fitoseídeo

Cerca de 150 espécies são hoje conhecidas no gênero Euseius, e encontradas principalmente nas regiões tropicais e subtropicais, especialmente em plantas arbóreas (Moraes et al 2004). A maioria das espécies de Euseius estudadas tem demonstrado preferência por pólen como fonte de alimento (McMurtry \& Croft 1997). A morfologia das quelíceras dos fitoseídeos está relacionada com seu hábito alimentar (Flechtmann \& McMurtry 1992a). Ácaros do gênero Euseius apresentam quelíceras curtas, com uma membrana distal ao dígito fixo e com sulco no deutosternum mais largo que outros fitoseídeos. Essas adaptações aparentemente possibilitam que os ácaros, ao perfurarem grãos de pólen, alimentem-se do conteúdo que deles escorre pela quelícera e penetra no sulco do deutosternum, sendo succionado pela cavidade oral (Flechtmann \& McMurtry 1992b, Flechtmann et al 1994). Apesar disso, eles também são capazes de se alimentar de presas, sobretudo das famílias Eriophyidae, Tarsonemidae, Tenuipalpidae e Tetranychidae (McMurtry \& Croft 1997). 
Alguns estudos têm demonstrado que certas espécies de Euseius podem se desenvolver e reproduzir alimentandose apenas de eriofídeos (Dicke et al 1990, Schausberger 1992).

Euseius alatus DeLeon é uma das seis espécies de fitoseídeos mais comumente encontradas em coqueiro (Cocos nucifera) no Nordeste brasileiro (Lawson-Balagbo et al 2008). A espécie é encontrada predominantemente em folhas (Gondim Jr \& Moraes 2001), mas ocasionalmente também é coletado em frutos infestados por Aceria guerreronis Keifer (Reis et al 2008). As dimensões relativamente grandes do predador dificultam sua penetração abaixo das brácteas dos frutos, onde se encontra A. guerreronis; a distância entre a superfície do fruto e a superfície ventral das brácteas pode variar de $75 \mu \mathrm{m}$ a $99 \mu \mathrm{m}$, dependendo da variedade (Aratchige 2007). Apesar dessa restrição morfológica no acesso à região meristemática dos frutos, é concebível que o predador possa atuar como inimigo natural de A. guerreronis durante sua fase de dispersão sobre o fruto e folhas, onde o predador é frequentemente encontrado.

Contudo, a comprovação dessa hipótese exige primeiramente a confirmação de que E. alatus pode utilizar A. guerreronis como fonte de alimento, permitindo-lhe se desenvolver e reproduzir. O objetivo deste trabalho foi avaliar, em condições de laboratório, a capacidade de $E$. alatus em se desenvolver e reproduzir, tendo apenas $A$. guerreronis como dieta, em comparação com outras dietas, incluindo pólen, considerado alimento muito adequado às espécies de Euseius.

\section{Material e Métodos}

Obtenção e criação de E. alatus. Os ácaros foram coletados em folhas de coqueiro, na Universidade Federal Rural de Pernambuco (UFRPE) $\left(8^{\circ} 01^{\prime}\right.$ 'S e $\left.34^{\circ} 56^{\prime} \mathrm{W}\right)$. No laboratório, os ácaros foram transferidos para arenas de criação, conforme descrito por Galvão et al (2007). As arenas foram constituídas por uma folha de feijão de porco (Canavalia ensiformes), com a face abaxial voltada para cima, sobreposta a um disco de papel de filtro sobre outro de espuma de polietileno, com $14 \mathrm{~cm}$ de diâmetro e $1 \mathrm{~cm}$ de espessura. Os discos foram colocados no interior de uma bandeja plástica e circundados com algodão hidrófilo umedecido com água destilada para evitar a fuga dos ácaros. Foram instaladas três unidades de criação, iniciadas com aproximadamente 100 ácaros cada, e mantidas com as seguintes dietas: (i) pólen de mamoneira (Ricinus communis), fêmeas adultas de Tetranychus urticae Koch (Acari: Tetranychidae) e mel a 10\%; (ii) somente pólen de mamoneira; e (iii) todos os estágios de A. guerreronis. $\mathrm{O}$ alimento foi reposto nas unidades a cada dois dias. $\mathrm{O}$ eriofídeo foi oferecido em pedaços da epiderme do fruto de aproximadamente $4 \times 4 \mathrm{~mm}$, contendo ácaros de todas as fases de desenvolvimento. Para dieta contendo T. urticae, foram oferecidas fêmeas com auxílio de pincel. O mel foi oferecido umedecendo-se um pedaço de papel toalha de $8 \mathrm{x}$ $8 \mathrm{~mm}$ colocado sobre uma lamínula de $18 \times 18 \mathrm{~mm}$. O pólen foi oferecido sobre uma lamínula de mesmo tamanho. As criações foram mantidas em incubadora a $27^{\circ} \mathrm{C}, 70 \pm 10 \%$ UR e $12 \mathrm{~h}$ de fotofase. As populações foram mantidas nas dietas durante 60 dias, antes da utilização dos ácaros para os experimentos.

Desenvolvimento e reprodução. Oitenta fêmeas adultas de E. alatus foram transferidas de cada unidade de criação para novas unidades, semelhantes às descritas anteriormente. Após $8 \mathrm{~h}$ de confinamento, as fêmeas foram retiradas e os ovos obtidos passaram a ser observados a cada $8 \mathrm{~h}$ para determinar o período de incubação e a viabilidade. Após a eclosão, as larvas foram individualizadas em novas unidades de criação, semelhantes às descritas anteriormente, mas com dimensões de $4 \times 4 \mathrm{~cm}$. As observações continuaram a cada $8 \mathrm{~h}$, para determinar a duração dos outros estágios de desenvolvimento e suas respectivas viabilidades. Após a emergência dos adultos, os machos foram isolados para determinação da longevidade, enquanto as fêmeas foram imediatamente acasaladas com machos obtidos das unidades de criação, avaliando-se os parâmetros de reprodução. Os machos que morriam eram substituídos por outros até a morte da fêmea. Os ovos obtidos nos dez primeiros dias foram isolados e os indivíduos obtidos criados até a fase adulta para determinação da razão sexual. O experimento foi realizado em incubadora a $27^{\circ} \mathrm{C}, 70 \pm 10 \%$ UR e $12 \mathrm{~h}$ de fotofase.

Utilizou-se o delineamento experimental inteiramente casualizado, com três tratamentos, cada um correspondendo a cada dieta. Os resultados foram submetidos à análise de variância, sendo as médias comparadas pelo Teste de Tukey $(\mathrm{P}=0,05)$, utilizando o programa computacional SANEST 3.0 (Zonta et al 1986).

Tabela de vida de fertilidade de $\boldsymbol{E}$. alatus. Os parâmetros da tabela de vida de fertilidade de E. alatus [taxa líquida de reprodução $\left(\mathrm{R}_{\mathrm{o}}\right)$, tempo médio da geração $(\mathrm{T})$, taxa intrínseca de crescimento populacional $\left(\mathrm{r}_{\mathrm{m}}\right)$, taxa finita de crescimento populacional $(\lambda)$ e tempo médio para a população dobrar em número (TD)] foram estimados através do pacote estatístico SAS (SAS Institute 1999-2001), adaptando o programa escrito por Maia et al (2000), o qual utiliza o método "Jackknife" para estimar intervalos de confiança das médias dos tratamentos e permite comparações entre pares de tratamentos empregandose o teste t.

\section{Resultados e Discussão}

As durações da fase de ovo, larva, protoninfa e deutoninfa foram mais longas quando $E$. alatus foi alimentada com pólen e com A. guerreronis $\left(F_{2,90}=43,47, \mathrm{P}=0,0001 ; F_{2,90}=16,79\right.$, $\left.\mathrm{P}=0,0001 ; F_{2,90}=7,08, \overrightarrow{\mathrm{P}}=0,0011 ; F_{2,90}=14,42, \overrightarrow{\mathrm{P}}=0,0001\right)$ se comparado a alimentação com T. urticae + pólen + mel, não havendo diferença entre as dietas a base de pólen e $A$. guerreronis (Tabela 1). Não houve diferença significativa entre as sobrevivências de E. alatus em cada um dos estágios de desenvolvimento isoladamente e em toda a fase imatura (P $>0,05$ ). A duração total da fase imatura (ovo a adulto) diferiu entre as três dietas, sendo menor em T. urticae + pólen + mel e maior quando alimentado apenas com $A$. guereronis $\left(F_{2}\right.$ $\left.{ }_{90}=65,79 ; \mathrm{P}=0,0001\right)$. Durante a manutenção das criações em laboratório, a população do predador desenvolveu-se mais lentamente quando alimentado exclusivamente $\operatorname{com} A$. 
Tabela 1 Duração média em dias ( $\pm \mathrm{EP})$ e sobrevivência (\%) dos estágios de desenvolvimento de Euseius alatus em diferentes dietas. Temp. $27^{\circ} \mathrm{C}$, UR $70 \pm 10 \%$ e fotofase de $12 \mathrm{~h}$.

\begin{tabular}{lcrrr}
\hline Estágio & Parâmetro & $\begin{array}{r}\text { T. urticae }+ \text { pólen } R \text {. communis }+ \\
\text { mel }\left(\mathrm{n}^{1}=73\right)\end{array}$ & $\begin{array}{r}\text { Pólen } \text { R. communis } \\
(\mathrm{n}=66)\end{array}$ & $\begin{array}{r}\text { A. guerreronis } \\
(\mathrm{n}=67)\end{array}$ \\
\hline \multirow{2}{*}{ Ovo } & Duração & $1,7 \pm 0,02 \mathrm{~b}$ & $2,0 \pm 0,04 \mathrm{a}$ & $2,0 \pm 0,05 \mathrm{a}$ \\
& Sobrevivência & $98,6 \pm 1,49 \mathrm{a}$ & $96,9 \pm 2,12 \mathrm{a}$ & $97,0 \pm 2,09 \mathrm{a}$ \\
Larva & Duração & $1,0 \pm 0,01 \mathrm{~b}$ & $1,1 \pm 0,03 \mathrm{a}$ & $1,2 \pm 0,04 \mathrm{a}$ \\
& Sobrevivência & $98,5 \pm 1,44 \mathrm{a}$ & $98,4 \pm 1,53 \mathrm{a}$ & $96,9 \pm 2,12 \mathrm{a}$ \\
Protoninfa & Duração & $1,1 \pm 0,02 \mathrm{~b}$ & $1,3 \pm 0,05 \mathrm{a}$ & $1,4 \pm 0,06 \mathrm{a}$ \\
& Sobrevivência & $98,5 \pm 1,45 \mathrm{a}$ & $96,8 \pm 2,17 \mathrm{a}$ & $96,8 \pm 2,15 \mathrm{a}$ \\
Deutoninfa & Duração & $1,2 \pm 0,02 \mathrm{~b}$ & $1,4 \pm 0,05 \mathrm{a}$ & $1,5 \pm 0,06 \mathrm{a}$ \\
& Sobrevivência & $97,0 \pm 2,05 \mathrm{a}$ & $96,7 \pm 2,21 \mathrm{a}$ & $96,7 \pm 2,19 \mathrm{a}$ \\
Ovo-adulto & Duração & $4,9 \pm 0,05 \mathrm{c}$ & $5,8 \pm 0,08 \mathrm{~b}$ & $6,2 \pm 0,11 \mathrm{a}$ \\
& Sobrevivência & $92,9 \pm 3,10 \mathrm{a}$ & $89,4 \pm 3,81 \mathrm{a}$ & $88,0 \pm 3,99 \mathrm{a}$ \\
\hline
\end{tabular}

${ }^{1}$ Número de observações

Médias seguidas de mesma letra, na linha, não diferem entre si pelo teste de Tukey $(\mathrm{P}>0,05)$.

guerreronis, embora a mortalidade de formas imaturas tenha sido semelhante entre as diferentes dietas.

Não se observou diferença entre as durações do período de pré-oviposição de E. alatus alimentada com as diferentes dietas $(\mathrm{P}>0,05)$. O período de oviposição foi maior na dieta $T$. urticae + pólen + mel e menor em pólen; a dieta à base de $A$. guerreronis promoveu duração intermediária $\left(F_{2,90}=4,09 ; \mathrm{P}=0,0200\right)$. O período de pós-oviposição foi maior quando o alimento foi T. urticae + pólen $+\operatorname{mel}\left(F_{290}\right.$ $=17,34 ; \mathrm{P}=0,0001)$. A longevidade de fêmeas foi maior em ácaros alimentados com T. urticae + pólen + mel do que daquelas alimentadas com pólen $\left(F_{2,90}=6,76 ; \mathrm{P}=0,0018\right)$. A fecundidade total diferiu entre os três tratamentos, sendo maior com T. urticae + pólen + mel e menor com apenas pólen $\left(F_{2,90}=17,60 ; \mathrm{P}=0,0001\right)$, enquanto a fecundidade diária e a razão sexual não difereriram $(\mathrm{P}>0,05)$ entre as dietas testadas (Tabela 2).

O período de oviposição de E. alatus variou 1 a 26,1 a 25 e 1 a 26 dias, registrando picos de produção de descendentes fêmeas no $5^{\circ}, 6^{\circ}$ e $7^{\circ}$ dias, com sobrevivência de $50 \%$ das fêmeas aos 26, 26 e 29 dias, respectivamente, para as dietas constituídas de A. guerreronis, pólen e T. urticae + pólen + mel (Figs 1 e 2).

O tempo médio da geração $(\mathrm{T})$, a taxa intrínseca de crescimento populacional $\left(\mathrm{r}_{\mathrm{m}}\right)$ e a taxa finita de crescimento populacional $(\lambda)$ não diferiram entre as três dietas testadas. Entretanto, o número de fêmeas produzidas, representado pela taxa líquida de reprodução $\left(\mathrm{R}_{\mathrm{o}}\right)$, foi maior quando $E$. alatus foi alimentada com T. urticae + pólen + mel ou apenas com A. guerreronis. No entanto, o valor obtido nessa última dieta não diferiu daquele obtido apenas com pólen. Como consequência, o tempo médio para a população dobrar em número (TD) apresentou resultado inverso à taxa líquida de reprodução $\left(\mathrm{R}_{\mathrm{o}}\right)$ (Tabela 3$)$.

Os valores estimados da tabela de vida de fertilidade para E. alatus nas três dietas testadas foram muito próximos àqueles encontrados por Reis \& Alves (1997), criando o predador em pólen de $R$. communis a $25^{\circ} \mathrm{C}\left(\mathrm{r}_{\mathrm{m}}=0,21 ; \lambda=\right.$ 1,$\left.2 ; \mathrm{TD}=3,2 ; \mathrm{R}_{\mathrm{o}}=21,4 ; \mathrm{T}=14,1\right)$.

A comparação de parâmetros como sobrevivência, fecundidade total, $\mathrm{R}_{0}, \mathrm{r}_{\mathrm{m}}$ e $\lambda$ entre a dieta composta por T. urticae + pólen + mel e a dieta constituída apenas por

Tabela 2 Duração média ( \pm EP), em dias, dos períodos de pré-oviposição, oviposição e pós-oviposição, fecundidade e longevidade de fêmeas de Euseius alatus e razão sexual em diferentes dietas. Temp. $27^{\circ} \mathrm{C}$, UR $70 \pm 10 \%$ e fotofase de $12 \mathrm{~h}$.

\begin{tabular}{|c|c|c|c|}
\hline Parâmetros biológicos & $\begin{array}{l}\text { T. urticae + pólen R. communis }+ \\
\text { mel }\left(\mathrm{n}^{1}=28\right)\end{array}$ & Pólen $R$. communis $(\mathrm{n}=31)$ & $\begin{array}{l}\text { A. guerreronis } \\
(\mathrm{n}=34)\end{array}$ \\
\hline Pré-oviposição & $1,6 \pm 0,13 \mathrm{a}$ & $1,9 \pm 0,12 \mathrm{a}$ & $1,9 \pm 0,11 \mathrm{a}$ \\
\hline Oviposição & $22,7 \pm 0,60 \mathrm{a}$ & $20,9 \pm 0,36 \mathrm{~b}$ & $22,0 \pm 0,36 \mathrm{ab}$ \\
\hline Pós-oviposição & $3,6 \pm 0,21 \mathrm{a}$ & $2,5 \pm 0,13 b$ & $2,4 \pm 0,14 b$ \\
\hline Fecundidade total & $37,9 \pm 0,58 \mathrm{a}$ & $33,5 \pm 0,52 \mathrm{c}$ & $36,0 \pm 0,42 b$ \\
\hline Fecundidade diária & $1,4 \pm 0,02 \mathrm{a}$ & $1,3 \pm 0,04 \mathrm{a}$ & $1,4 \pm 0,05 \mathrm{a}$ \\
\hline Longevidade $q$ & $28,0 \pm 0,70 \mathrm{a}$ & $25,3 \pm 0,40 \mathrm{~b}$ & $26,3 \pm 0,39 a b$ \\
\hline Razão sexual & $0,7 \pm 0,00 \mathrm{a}$ & $0,7 \pm 0,00 \mathrm{a}$ & $0,7 \pm 0,00 \mathrm{a}$ \\
\hline
\end{tabular}

${ }^{1}$ Número de observações

Médias seguidas da mesma letra, na linha, não diferem entre si, pelo teste de Tukey $(\mathrm{P}>0,05)$. 
A. guerreronis, indica que E. alatus pode se alimentar do eriofídeo na cultura do coqueiro, embora não co-habite a região meristemática do fruto com a presa. Segundo Fernando

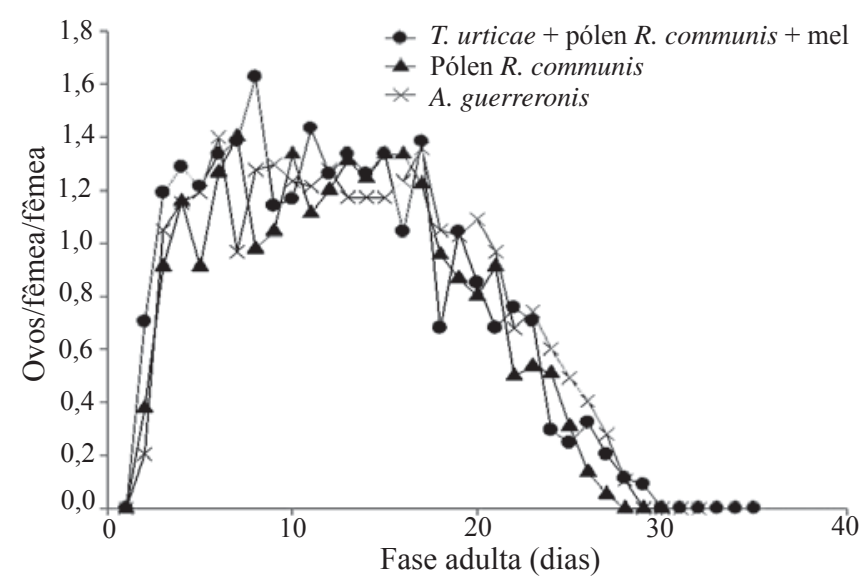

Fig 1 Ritmo de produção de descendentes fêmeas de Euseius alatus em diferentes dietas. Temp. $27^{\circ} \mathrm{C}$, UR $70 \pm 10 \%$ e fotofase de $12 \mathrm{~h}$.

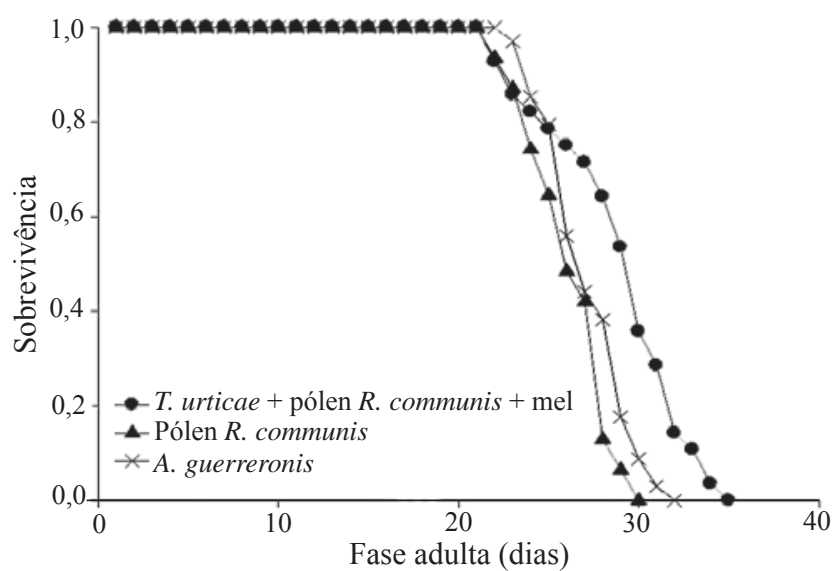

Fig 2 Sobrevivência de fêmeas de Euseius alatus em diferentes dietas. Temp. $27^{\circ} \mathrm{C}$, UR $70 \pm 10 \%$ e fotofase de $12 \mathrm{~h}$. et al (2003) a população de A. guerreronis concentrase no coqueiro, entre o segundo e o sexto cacho, após a inflorescência totalmente aberta. A infestação inicia-se, normalmente, no segundo cacho, atingindo pico populacional no quinto cacho, reduzindo drasticamente em direção aos cachos mais velhos. De acordo com Moore \& Alexandre (1987), A. guerreronis apresenta fototropismo negativo, não sendo encontrada na superfície do fruto durante o dia. Contudo, segundo Moore \& Howard (1996), durante a noite A. guerreronis provavelmente sai da região meristemática de frutos mais velhos, inadequados nutricionalmente ou altamente infestados, para migrar para frutos mais novos. Nessa ocasião, E. alatus poderia predar A. guerreronis, colaborando para o controle biológico do eriofídeo.

Diversos eriofídeos também são relatados na cultura do coqueiro no Brasil, sendo alguns frequentemente encontrados sobre os frutos, como Amrineus cocofolius Flechtmann, e outros sobre folhas, como Retracrus johnstoni Keifer, e um complexo de espécies de Notostrix (Navia et al 2007). Portanto, além de A. guerreronis, os fitoseídeos associados ao coqueiro, como E. alatus, devem estar em contato direto com várias espécies de eriofídeos e, provavelmente, alimentam-se facultativamente deste grupo de ácaros. O desenvolvimento e a reprodução de E. alatus apresentados neste trabalho indicam que $A$. guerreronis é um alimento adequado àquele predador.

Apesar de A. guerreronis ter permitido o desenvolvimento e a reprodução adequada de E. alatus, são necessários estudos adicionais sobre fatores ecológicos que permitam melhor avaliar o papel desempenhado pelo predador no campo. Alguns dos principais fatores a serem avaliados referem-se à preferência alimentar do predador na presença de diferentes opções (como certamente ocorre no campo), ao sincronismo entre os períodos diários de maior atividade do predador e da dispersão da praga e à capacidade de busca do predador.

\section{Agradecimentos}

Ao CNPq pela concessão da bolsa de iniciação científica ao primeiro autor, junto à Universidade Federal Rural

Tabela 3 Parâmetros da tabela de vida de fertilidade registrados para Euseius alatus em três dietas alimentares. Taxa líquida de reprodução $\left(\mathrm{R}_{\mathrm{o}}\right)$, tempo médio de geração $(\mathrm{T})$, taxa intrínseca de crescimento populacional $\left(\mathrm{r}_{\mathrm{m}}\right)$, taxa finita de crescimento populacional $(\lambda)$ e tempo médio em dias para duplicar a população em números (TD). Temp. $27^{\circ} \mathrm{C}$, UR $70 \pm$ $10 \%$ e fotofase de $12 \mathrm{~h}$.

\begin{tabular}{|c|c|c|c|c|c|}
\hline Dieta & $\begin{array}{c}\mathrm{R}_{\mathrm{o}} \\
(q)(q)^{-1}\end{array}$ & $\begin{array}{c}\mathrm{T} \\
\text { (dias) }\end{array}$ & $\begin{array}{c}\mathrm{r}_{\mathrm{m}} \\
(+)(+)^{-1}(\mathrm{dia})^{-1}\end{array}$ & $\lambda$ & $\begin{array}{c}\text { TD } \\
\text { (dias) }\end{array}$ \\
\hline $\begin{array}{l}\text { T. urticae }+ \\
\text { pólen } R \text {. communis }+ \text { mel }\end{array}$ & $\begin{array}{c}23,91 \mathrm{a} \\
(23,14-24,70)^{1}\end{array}$ & $\begin{array}{c}14,32 \mathrm{a} \\
(13,85-14,79)\end{array}$ & $\begin{array}{c}0,22 \mathrm{a} \\
(0,21-0,22)\end{array}$ & $\begin{array}{c}1,24 \mathrm{a} \\
(1,23-1,25)\end{array}$ & $\begin{array}{c}3,12 \mathrm{~b} \\
(3,01-3,23)\end{array}$ \\
\hline A. guerreronis & $\begin{array}{c}22,19 \mathrm{ab} \\
(21,66-22,72)\end{array}$ & $\begin{array}{c}14,20 \mathrm{a} \\
(13,78-14,62)\end{array}$ & $\begin{array}{c}0,21 \mathrm{a} \\
(0,21-0,22)\end{array}$ & $\begin{array}{c}1,24 \mathrm{a} \\
(1,23-1,25)\end{array}$ & $\begin{array}{c}3,17 \mathrm{ab} \\
(3,09-3,25)\end{array}$ \\
\hline Pólen R. communis & $\begin{array}{c}20,97 \mathrm{~b} \\
(20,03-21,43)\end{array}$ & $\begin{array}{c}14,55 \mathrm{a} \\
(14,21-14,90)\end{array}$ & $\begin{array}{c}0,20 \mathrm{a} \\
(0,20-0,21)\end{array}$ & $\begin{array}{c}1,23 \mathrm{a} \\
(1,22-1,23)\end{array}$ & $\begin{array}{c}3,31 \mathrm{a} \\
(3,25-3,37)\end{array}$ \\
\hline
\end{tabular}

${ }^{1}$ Intervalo de confiança a $95 \%$ de probabilidade.

Médias seguidas pela mesma letra, na coluna, não diferem entre si por meio de comparações de tratamentos dois a dois, através do intervalo de confiança a 95\% de probabilidade após estimativa de erros pelo método Jackknife (SAS Institute 1999-2001). 
de Pernambuco, e também pela concessão de bolsa de produtividade em pesquisa para o terceiro e quarto autores.

\section{Referências}

Aratchige N S (2007) Predators and the accessibility of herbivore refuges in plants. Amsterdam, Academisch Proefschrift, 124p.

Dicke M, Sabelis M W, De Jong M (1990) Do phytoseiids select the best prey species in terms of reproductive success? Exp Appl Acarol 8: 161-73.

Fernando L C P, Aratchige N S, Peris T S G (2003) Distribution patterns of coconut mite, Aceria guerreronis, and its predator Neoseiulus aff. paspalivorus in coconut palms. Exp Appl Acarol 31: 71-78.

Flechtmann C H W, Evans G O, McMurtry J A (1994) Some noteworthy features of the chelicerae and subcapitulum of Phytoseiulus longipes Evans (Acari: Mesostigmata: Phytoseiidae), with observations on the preoral channel in the Phytoseiidae. Exp Appl Acarol 18: 293-299.

Flechtmann C H W, McMurtry J A (1992a) Studies on how phytoseiid mites feed on spider mites and pollen. Int J Acarol 18: $157-162$

Flechtmann C H W, McMurtry J A (1992b) Studies of cheliceral and deutosternal morphology of some Phytoseiidae (Acari: Mesostigmata) by scanning electron microscopy. Int J Acarol 18: 163-169.

Galvão A S, Gondim Jr M G C, Moraes G J, Oliveira J V de (2007) Biologia de Amblyseius largoensis (Muma) (Acari: Phytoseiidae), um potencial predador de Aceria guerreronis Keifer (Acari: Eriophyidae) em coqueiro. Neotrop Entomol 36: 465-470.

Gondim Jr M G C, Moraes G J (2001) Phytoseiid mites (Acari: Phytoseiidae) associated with palm trees (Arecaceae) in Brazil. Syst Appl Acarol 6: 65-94.

Lawson-Balagbo L M, Gondim Jr M G C, Moraes G J, Hanna R, Schausberger P (2008) Exploration of the acarine fauna on coconut palm in Brazil with emphasis on Aceria guerreronis (Acari: Eriophyidae) and its natural enemies. Bull Entomol Res 97: 83-96.
Maia A H N, Luiz A J B, Campanhola C (2000) Statistical inference on associated fertility life table parameters using Jackknife technique: computational aspects. J Econ Entomol 93: 511-518.

McMurtry J A, Croft B A (1997) Life styles of phytoseiid mites and their roles in biological control. Annu Rev Entomol 42: 291-321.

Moore D, Alexander L (1987) Aspects of migration and colonization of the coconut palm by the coconut mite, Eriophyes guerreronis (Keifer) (Acari: Eriophyidae). Bull Entomol Res 77: 641-650.

Moore D, Howard F W (1996) Coconuts, p. 561-570. In Lindquist E E, Sabelis M W, Bruin J (eds) Eriophyoid mites: their biology, natural enemies and control. Amsterdam, Elsevier, 790p.

Moraes G J, McMurtry J A, Denmark H A, Campos C B (2004) A revised catalog of the mite family Phytoseiidae. Zootaxa 434: $1-494$.

Navia D, Gondim Jr M G C, Moraes G J (2007) Eriophyoid mites (Acari: Eriophyoidea) associated with palm trees. Zootaxa 1389:1-30.

Reis A C, Gondim Jr M G C, Moraes G J, Hanna R, Schausberger P, Lawson-Balagbo L E, Barros R (2008) Population dynamics of Aceria guerreronis Keifer (Acari: Eriophyidae) and associated predators on coconut fruits in Northeastern Brazil. Neotrop Entomol 37: 457-462.

Reis P R, Alves E B (1997) Biologia do ácaro predador Euseius alatus DeLeon (Acari: Phytoseiidae). An Soc Entomol Bras 26: 359-361.

SAS Institute (1999-2001) SAS/STAT ${ }^{\circledR}$ User's guide, version 8.02, TS level 2MO. SAS Institute Inc. Cary, NC.

Schausberger P (1992) Vergleichende Untersuchungen über den Einfluss unterschiedlicher Nahrung auf die präimaginalentwicklung und die Reproduktion von Amblyseius aberrans Oud. und Amblyseius finlandicus Oud. (Acarina: Phytoseiidae). J Appl Entomol 113:476-86.

Zonta E P, Silveira P, Machado A A (1986) Sistema de análise estatística (SANEST 3.0). Pelotas, Instituto de Física e Matemática, UFPel, 399p.

Received 12/IX/07. Accepted 06/XI/08. 